\title{
Effect of Aging on the Progression of Articular Cartilage Damage in a Rat Osteoarthritis Model
}

\author{
Masanori Watanabe ${ }^{1,}$, , Satoshi Kojima ${ }^{2}$, Keiji Asada ${ }^{3}$, Itaru Hibino ${ }^{1}$, Masahiro Hoso ${ }^{4}$ \\ ${ }^{1}$ Department of Physical Therapy, Faculty of Rehabilitation Science, Nagoya Gakuin University, Seto, Japan \\ ${ }^{2}$ Course of Rehabilitation, Graduate School of Rehabilitation, Kinjo University, Hakusan, Japan \\ ${ }^{3}$ Department of Physiotherapy, Faculty of Health Science, Suzuka University of Medical Science, Suzuka, Japan \\ ${ }^{4}$ Division of Health Sciences, Graduate School of Medical Science, Kanazawa University, Kanazawa, Japan
}

Email address:

m.wtnb@ngu.ac.jp (M. Watanabe)

${ }^{*}$ Corresponding author

To cite this article:

Masanori Watanabe, Satoshi Kojima, Keiji Asada, Itaru Hibino, Masahiro Hoso. Effect of Aging on the Progression of Articular Cartilage Damage in a Rat Osteoarthritis Model. International Journal of Clinical and Experimental Medical Sciences.

Vol. 4, No. 2, 2018, pp. 21-25. doi: 10.11648/j.ijcems.20180402.11

Received: March 28, 2018; Accepted: April 17, 2018; Published: May 10, 2018

\begin{abstract}
This study aimed to investigate and clarify the effect of aging on the progression of cartilage damage using a rat osteoarthritis model. In total, 12 rats, including three rats per age group, were assigned to the experimental group, and two rats (one 3 and 18 months old each) were assigned to the control group. In the experimental group, joint instability was induced using the destabilization of the medial meniscus model. After completion of the 4-week experimental period, the bilateral knee joints were harvested and the histological changes in the articular structures of the joints were observed using a light microscope. Medial dislocation of the medial meniscus, low staining of the cartilaginous substrate on the tibial side localized around the medial meniscus tip, and irregularities and fibrillation of the superficial layer of the cartilage on the tibial side were observed in all experimental age groups. No significant age-related differences were observed in the degree of observed findings and the score of cartilage degeneration. The results indicate that aging does not affect the early stages of progression of cartilage damage.
\end{abstract}

Keywords: Osteoarthritis, Aging, Histopathology

\section{Introduction}

Osteoarthritis (OA) is one of the most common degenerative joint disease that mainly affects the articular cartilage and is associated with aging. It mostly affects the joints that have been under continuous stress and overused such as the hip, knee, fingers, and lower spine. An estimated $9.6 \%$ of men and $18.0 \%$ of women aged $>60$ years worldwide have symptomatic OA [1-2]. Of patients with OA, $80 \%$ have limitations in movement and $25 \%$ cannot perform their major activities of daily living. A previous large-scale, population-based cohort study named research on osteoarthritis/osteoporosis against disability investigated the prevalence of knee osteoarthritis in the Japanese population and reported that $25,300,000$ people $(8,600,000$ men and $16,700,000$ women) aged $>40$ years might be affected [3]. In the Comprehensive Survey of
Living Conditions 2016 by the Ministry of Health, Labour and Welfare in Japan, osteoarthropathy [4] was reported as the number one cause of certification for the need of support in elderly people in the Long-Term Care Insurance Act. Some studies [5-6] have reported that knee injuries, such as meniscal lesions, increase joint instability and cause OA. It is assumed that the progression of cartilage damage after trauma is affected by aging because OA is associated with degeneration of the articular cartilage with aging [7-9]. However, the association between the progression of cartilage damage after trauma and the effects of aging on knee OA is unknown.

Animal models have helped a lot in understanding the pathophysiology of OA [10]. The animal models currently used in $\mathrm{OA}$ research are divided into induced and 
spontaneous models. According to the procedure used to induce OA, induced models are further subdivided into surgical and chemical models. Surgical procedures induce OA by producing joint instability and altering load-bearing. The degeneration of cartilage in OA is affected by differences in the surgical techniques used to produce instability in the knee joint [11]. The best-described and most frequently used surgical model for joint instability is the anterior cruciate ligament model (ACLT) [10]. However, several recent studies have used the destabilization of the medial meniscus (DMM) model [12-13]. Authors previously reported that the progression of cartilage damage after surgery is slower in the DMM model than in the ACLT model [14]. Thus, the DMM model is adequate for observing the early stages of cartilage damage in OA. In these animal models, it is predicted that cartilage degeneration due to aging accompanies or response to surgical technique is expected to differ. In addition, the age of the animal used for study is limited depending on the species. Few reports are available on the effects of aging on surgical models of joint instability.

This study aimed to use histopathological changes to investigate and clarify the effect of aging on the progression of cartilage damage in an animal OA model.

\section{Materials and Methods}

In total, 14 male Wistar rats were used: four 3-month-old (320-360 g), three 6-month-old (480-550 g), three 12-month-old (530-600 g), and four 18-month-old (550-660 g) rats. Twelve rats, including three rats per age group, were assigned to the experimental group, and two rats (one 3 and 18 month olds each) were assigned to the control group. The rats were kept individually in plastic cages in a 12-h light/dark cycle and allowed food and water ad libitum, with the room temperature set at $20^{\circ} \mathrm{C}-26^{\circ} \mathrm{C}$. This study was performed in accordance with the Regulations on Animal Experiments of Nagyoa Gakuin University and was approved by the Animal Experiments Committee of the university (approval number, 2013-001).

Following previous studies [12], a bilateral DMM model were established to the hind legs of the rats in the experimental group. With the rat under general anesthesia induced with isoflurane, the periphery of the knee joint was shaved, a longitudinal 15-20-mm incision was made on the medial side of the thigh, and the joint capsule was resected from the medial side of the patellar tendon. The patellar tendon was pulled laterally to identify the medial meniscotibial ligament and transected; the joint capsule and skin were closed by sutures. For subcutaneous and dermal sutures, 5-0 and 6-0 suturing threads (Akiyama Seisakusyo, Japan) were used, respectively. The rats in the experimental group were kept under normal conditions for the subsequent 4 weeks and those in the control group were kept under normal conditions without intervention.

After completion of the experimental period, perfusion fixation was performed with $4 \%$ paraformaldehyde, and the bilateral knee joints were harvested. Then, the joints were decalcified for $72 \mathrm{~h}$ in Plank Rychlo solution (Decalcifying Solution A, Wako Pure Chemical Industries) at $4{ }^{\circ} \mathrm{C}$. The knee joints were resected from the frontal plane close to the center of the anterior and posterior sides of the tibial bone. The joints were neutralized with $5 \%$ sodium sulfate and treated with paraffin after fat removal with alcohol. Using a sliding microtome (REM-710,

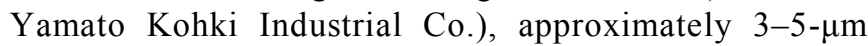
slices were obtained from the prepared paraffin block, and hematoxylin and eosin staining and safranin-O/fast green staining were performed. The slides were observed using a light microscope (BX-51, Olympus Corporation), and images were taken using a digital microscope camera (DP73, Olympus Corporation).

Knee joint observations were taken at two points on each leg $200 \mu \mathrm{m}$ apart near the center of the tibial bone. The degree of cartilage degeneration in the experimental group was graded by a researcher after masking according to the Osteoarthritis Research Society International cartilage histopathology assessment system [15]. The Kruskal-Wallis test was used to compare the scores; $p<0.05$ was considered significant. Statistical software R (2.8.1) was used for analysis.

\section{Results}

Medial dislocation of the medial meniscus (Figure 1, top), low staining of the cartilaginous substrate on the tibial side localized around the medial meniscus tip (Figure 1, middle), and irregularities and fibrillation of the superficial layer of the cartilage on the tibial side (Figure 1, bottom) were observed in all experimental age groups. No significant age-related differences were observed in the degree of the observed findings, with median cartilage degeneration scores of $6.0,3.0$, and 5.0 at 3,12 , and 18 months, respectively ( $p=$ 0.173) (Figure 2). 


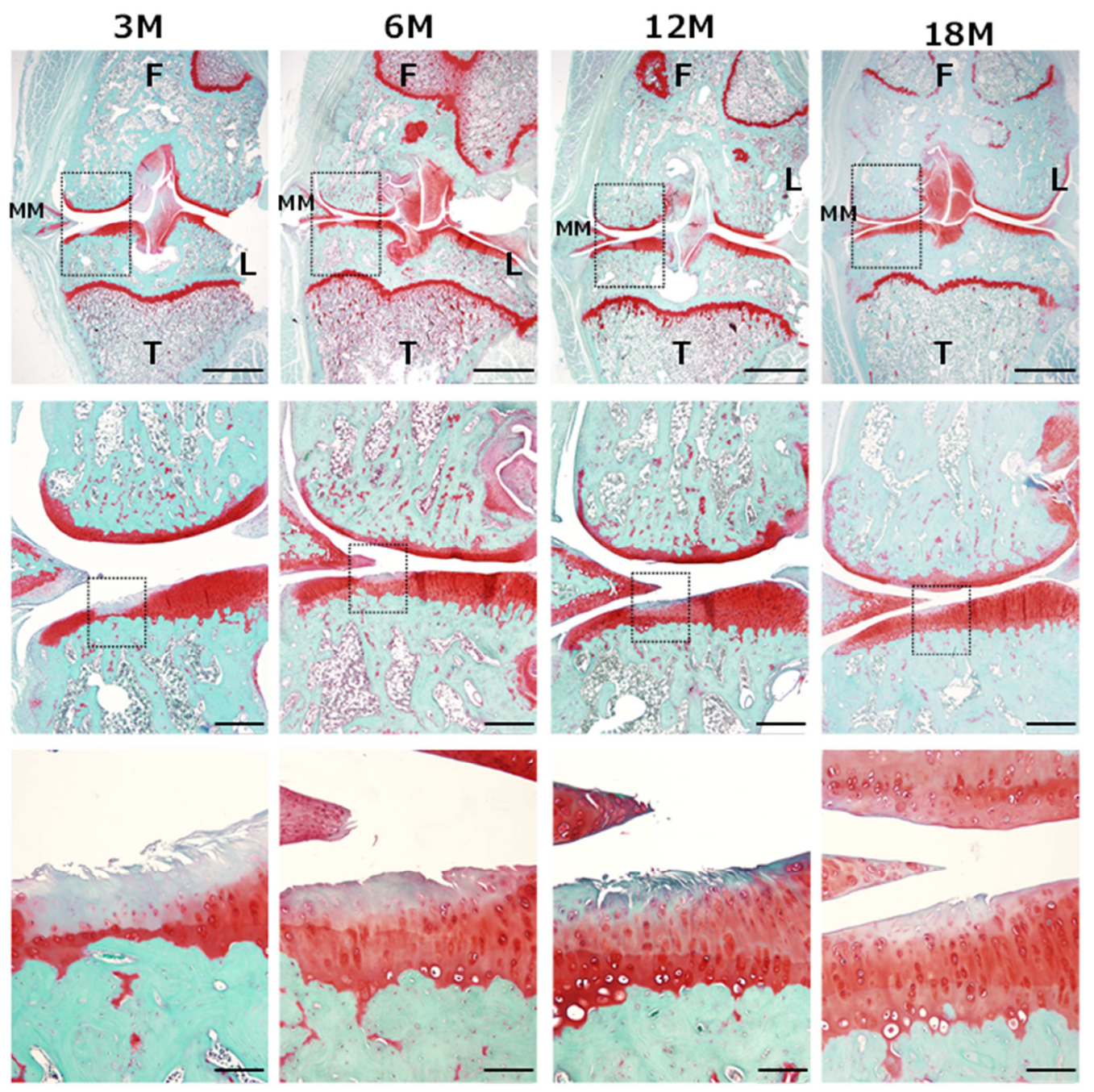

Figure 1. Joint cartilage findings among the experimental groups (safranin-O/fast green staining).

Middle and bottom images are magnified findings of the part indicated by the dotted lines. Top: Dislocation of the medial meniscus is observed at all ages. Scale bars $=2 \mathrm{~mm}$. Middle: Low staining of the cartilaginous substrate on the tibial bone localized around the tip of the medial meniscus. Scale bars $=500 \mu \mathrm{m}$. Bottom: Irregularities and fibrillation of the superficial layer of the cartilage. Scale bars $=100 \mu \mathrm{m}$. F, femur; T, tibia; MM, medial meniscus; $\mathrm{L}$, lateral.

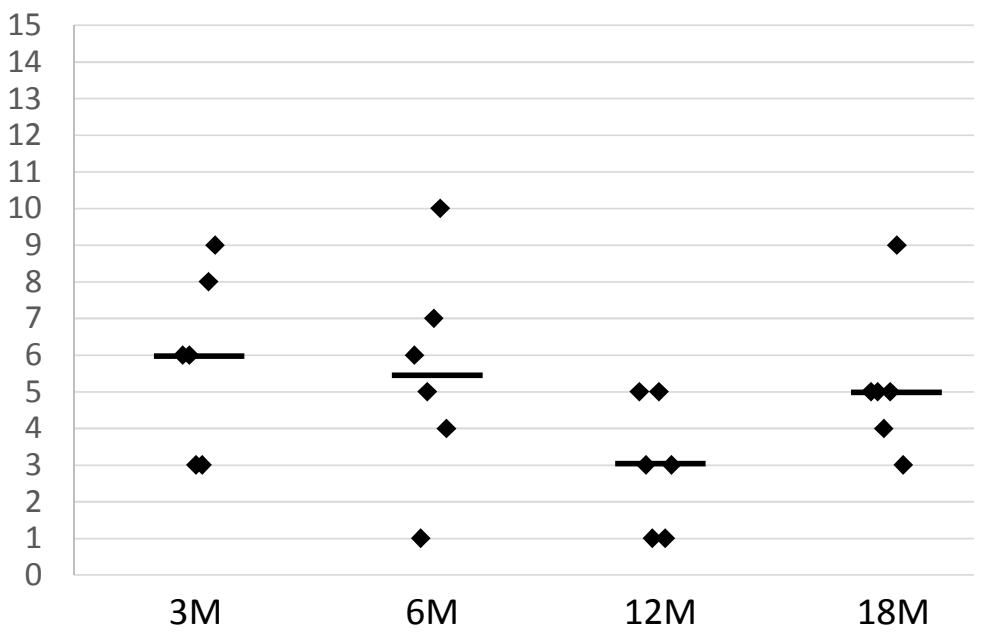

Figure 2. Joint cartilage degeneration scores among the experimental groups.

The degree of cartilage degeneration was graded by the Osteoarthritis Research Society International cartilage histopathology assessment system. Horizontal lines indicate median values. No significant differences were observed among the groups (Kruskal-Wallis test; $p>0.05$ ). 
No degenerative findings were observed in the articular cartilage of the control group, and no changes were observed between the age groups in weeks (Figure 3).

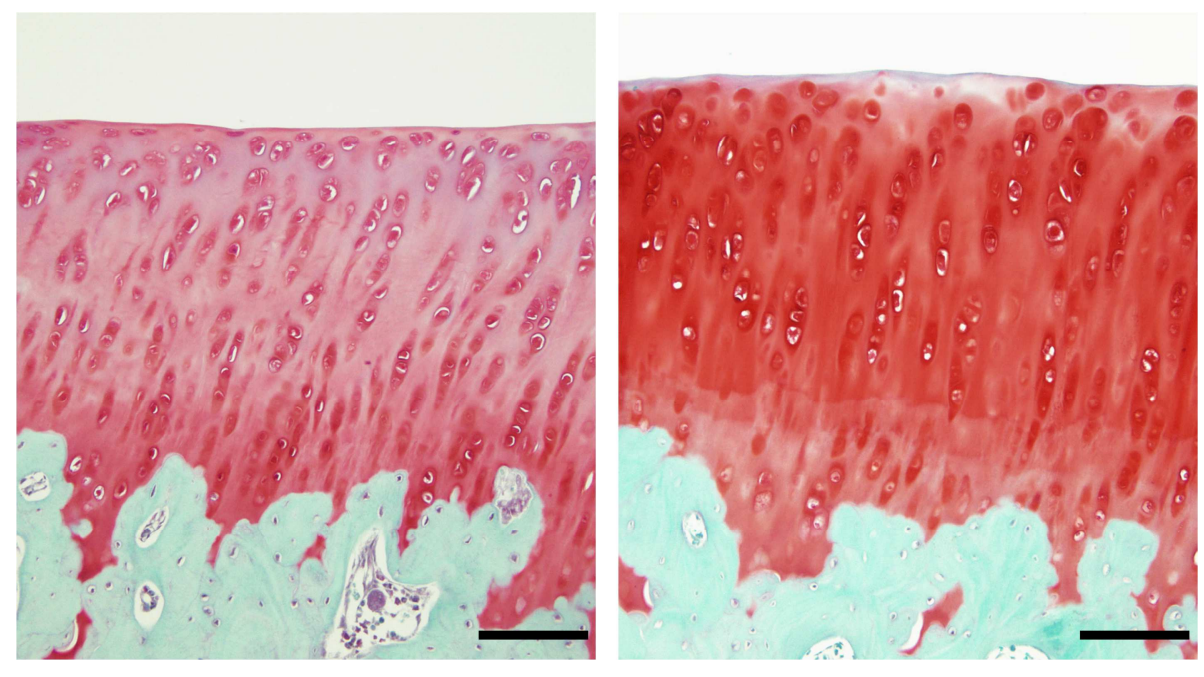

Figure 3. Joint cartilage findings in the control group (safranin-O/fast green staining).

Left, 3 months; right, 18 months. No degenerative findings were seen in the articular cartilage of the control group, and no changes were seen between the age groups in weeks. Scale bars $=100 \mu \mathrm{m}$. F, femur; T, tibia; MM, medial meniscus; L, lateral.

\section{Discussion}

The DMM model of OA using rats of various ages (in months) was established to compare the effects of aging on the degree of articular cartilage trauma in the early stages. The marked differences between the experimental age groups in the degree of cartilage trauma were not found.

No age-related differences were seen in the cartilage tissue findings in the control group, suggesting that age did not affect the joint cartilage trauma in the early stages of the DMM model, assuming that the condition of the cartilage before the surgical intervention was equal. Ferrándiz et al. [16] established an ACLT model in 2- and 12-month-old rats to compare cartilage degeneration scores between the two age groups 10 weeks later. They found no difference in degeneration scores between the two age groups of sham-operated rats; however, they found a significant increase (progressive destruction) in the 12-month-old rats compared with the 2-month-old rats in the intervention group. The authors concluded that aging affected the degree of response to a traumatic intervention. This study differed because of the following possible reasons. First, there may have been a difference between the postoperative periods of the OA models used in the two studies. The DMM model used in this study caused lesser knee instability than the ACLT model, and the degree of cartilage injury progression was milder. In addition, the postoperative period in this study was too short to show an age-related difference.

The present study has some limitations. First, the sample size was small, which is presumed to be one of the reasons why there were no significant differences between the histological findings of the age groups (in weeks). In particular, the number of rats in the control group (two rats) may have been insufficient for observing the differences in the degree of cartilage degeneration, which are believed to occur with aging. Moriyama et al. [17] observed knee joint histological findings of 4-month-old rats and reported that they observed OA-like degeneration such as fissures and clustering. The rats used in this study were judged sufficiently aged for the purposes of the experiment based on the fact that the rate of natural death increases after 18 months [18]; however, the 6-month age difference in the rats used in the previous study corresponds to a difference of 15 years in humans [19-20]. This age difference may have affected the findings of cartilage degeneration in the control group. If cartilage degeneration or the associated functional decline of the cartilage that was undetectable by the histological methods used in this study occurred in the aged rats, including the 18-month-old rats, it is possible that there was more notable cartilage degeneration in the young rats, suggesting that a larger sample size would be required for further validation. Furthermore, the amount of activity was not measured in the rats in this study. Thus, it remains unknown if there was a difference in the amount of activity in relation to aging. Although no similar reports have measured the relationship between the amount of activity and aging over time, it has been reported that there is a decline in the amount of exercise between young (3-4 months) and old (24 months) rats [21-22]. This suggests that changes in mechanical stress associated with the increase or decrease of activity can also affect the progression of cartilage degeneration. Further verification is warranted.

\section{Conclusion}

The results of this study showed that joint cartilage trauma in the early stages following DMM model surgery was not affected by aging if there were no differences in the cartilage histological images before the model intervention. 


\section{Acknowledgements}

All authors would like to deep thank to staff of Department of Human Pathology, Faculty of Medicine, Institute of Medical, Pharmaceutical and Health Sciences, Kanazawa University. This work was supported by JSPS KAKENHI Grant Number 16K01526 and a research grant in 2017 from Nagoya Gakuin University.

\section{Conflict of Interest Statement}

The authors have no conflicts of interest directly relevant to the content of this article.

\section{References}

[1] Woolf AD, Pfleger B. Burden of major musculoskeletal conditions. Bull World Health Organ. 2003; 81:646-56.

[2] WHO. Chronic Diseases and Health Promotion. http://www.who.int/chp/topics/rheumatic/en/(Accessed Jan. 11, 2018)

[3] Yoshimura N: Epidemiology of osteoarthritis in Japan; the ROAD study. Clin Calcium. 2011; 21:821-825 (in Japanese)

[4] Ministry of Health, Labour and Welfare. Comprehensive Survey of Living Conditions. http://www.mhlw.go.jp/toukei/saikin/hw/k-tyosa/k-tyosa16/in dex.html (Accessed Jan. 11, 2018)

[5] Badlani JT, Borrero C, Golla S, Harner CD, Irrgang JJ, et al.: The effects of meniscus injury on the development of knee osteoarthritis: data from the osteoarthritis initiative. Am J Sports Med. 2013; 41: 1238-1244.

[6] Englund M, Guermazi A, Roemer FW, et al.: Meniscal tear in knees without surgery and the development of radiographic osteoarthritis among middle-aged and elderly persons: The Multicenter Osteoarthritis Study. Arthritis Rheum. 2009; 60: 831-839.

[7] Rahmati M, Nalesso G, Mobasheri A, et al.: Aging and osteoarthritis: Central role of the extracellular matrix. Ageing Res Rev. 2017; 40: 20-30.

[8] Loeser RF: Age-related changes in the musculoskeletal system and the development of osteoarthritis. Clin Geriatr Med. 2010; 26: 371-386.

[9] Shane Anderson A, Loeser RF: Why is osteoarthritis an age-related disease? Best Pract Res Clin Rheumatol. 2010; 24: 15-26.
[10] Lampropoulou-Adamidou K, Lelovas P, Karadimas EV, et al.: Useful animal models for the research of osteoarthritis. Eur J Orthop Surg Traumatol. 2014; 24: 263-271.

[11] Kamekura S, Hoshi K, Shimoaka T, Chung U, et al.: Osteoarthritis development in novel experimental mouse models induced by knee joint instability. Osteoarthritis Cartilage. 2005; 13: 632-641.

[12] Galsson SS, Blanchet TJ, Morris EA: The surgical destabilization of the model meniscus (DMM) model of osteoarthritis in the $129 / \mathrm{SvEv}$ mouse. Osteoarthritis Cartilage. 2007; 15: 1061-1069.

[13] Glasson SS, Askew R, Sheppard B, et al.: Deletion of active ADAMTS5 prevents cartilage degradation in a murine model of osteoarthritis. Nature. 2005; 434: 644-648.

[14] Watanabe M, Kojima S, Asada K, et al.: Comparison the rat models of osteoarthritis for examining the effect of the physical therapy. Journal of Nagoya Gakuin University, Medical, Health, and Sports Sciences (in Japanese) (in press).

[15] Gerwin N, Bendele AM, Glasson S, et al.: The OARSI histopathology initiative - recommendations for histological assessments of osteoarthritis in the rat. Osteoarthritis Cartilage. 2010; 18: S24-34.

[16] Ferrándiz ML, Terencio MC, Ruhí R, et al.: Influence of age on osteoarthritis progression after anterior cruciate ligament transection in rats. Exp Gerontol. 2014; 55: 44-48.

[17] Moriyama H, Kanemura N, Brouns I, et al.: Effects of aging and exercise training on the histological and mechanical properties of articular structures in knee joints of male rat. Biogerontology. 2012; 13: 369-381.

[18] Kuramoto K, Shumiya S, Inoue T I, et al.: Pathological findings of the rats naturally died during aged animal rearing. Biomedical Gerontology. 1986; 10: 71-72 (in Japanese).

[19] Andreollo NA, Santos EF, Araújo MR, et al.: Rat's age versus human's age: what is the relationship? Arq Bras Cir Dig. 2012; 25: 49-51.

[20] Sengupta P.: The Laboratory Rat: Relating Its Age With Human's. Int J Prev Med. 2013; 4: 624-630.

[21] Barrientos RM, Watkins LR, Rudy JW, et al.: Characterization of the sickness response in young and aging rats following $\mathrm{E}$. coli infection. Brain Behav Immun. 2009; 23: 450-454.

[22] Boguszewski P, Zagrodzka J. Emotional changes related to age in rats--a behavioral analysis. Behav Brain Res. 2002; 133: 323-332. 